\title{
Prevalence and incidence of interstitial lung diseases in a multi-ethnic county of Greater Paris
}

\author{
Boris Duchemann ${ }^{1,2}$, Isabella Annesi-Maesano ${ }^{3}$, Camille Jacobe de Naurois ${ }^{4}$, \\ Shreosi Sanyal ${ }^{3}$, Pierre-Yves Brillet ${ }^{2,5}$, Michel Brauner $^{5}$, \\ Marianne Kambouchner ${ }^{6}$, Sophie Huynh ${ }^{7}$, Jean Marc Naccache ${ }^{8}$, \\ Raphael Borie ${ }^{9}$, Jacques Piquet ${ }^{10}$, Arsène Mekinian ${ }^{11}$, Jerôme Virally ${ }^{7}$, \\ Yurdagul Uzunhan ${ }^{1,2}$, Jacques Cadranel $^{8}$, Bruno Crestani ${ }^{9}$, Olivier Fain ${ }^{11}$, \\ Francois Lhote $^{12}$, Robin Dhote ${ }^{13}$, Nathalie Saidenberg-Kermanac' ${ }^{14}$, \\ Paul-André Rosental ${ }^{15}$, Dominique Valeyre ${ }^{1,2}$ and Hilario Nunes ${ }^{1,2}$
}

@ERSpublications

ILD prevalence reaches $97.9 / 100000$, with a fairly low prevalence of IPF and a high contribution of secondary ILDs http://ow.ly/vLXq30bssKR

Cite this article as: Duchemann B, Annesi-Maesano I, Jacobe de Naurois C, et al. Prevalence and incidence of interstitial lung diseases in a multi-ethnic county of Greater Paris. Eur Respir J 2017; 50: 1602419 [https://doi.org/10.1183/13993003.02419-2016].

ABSTRACT The objective of the study was to estimate the prevalence and incidence of interstitial lung diseases (ILDs) in Seine-Saint-Denis, a multi-ethnic county of Greater Paris, France.

Patients with ILDs were identified between January and December 2012 by using several sources; all potentially involved medical specialists from public and private hospitals, community-based pulmonologists and general practitioners, and the Social Security system. Diagnoses were validated centrally by an expert multidisciplinary discussion.

1170 ILD cases were reported (crude overall prevalence: $97.9 / 10^{5}$ and incidence: $19.4 / 10^{5} /$ year). In the 848 reviewed cases, the most prevalent diagnoses were sarcoidosis $(42.6 \%)$, connective tissue diseases associated ILDs (CTDs-ILDs) (16\%), idiopathic pulmonary fibrosis (IPF) (11.6\%), and occupational ILDs (5.0\%), which corresponded to a crude prevalence of $30.2 / 10^{5}$ for sarcoidosis, $12.1 / 10^{5}$ for CTDs-ILDs and $8.2 / 10^{5}$ for IPF. The prevalence of fibrotic idiopathic interstitial pneumonias, merging IPF, nonspecific interstitial pneumonia and cases registered with code J84.1 was $16.34 / 10^{5}$. An adjusted multinomial model demonstrated an increased risk of sarcoidosis in North Africans and Afro-Caribbeans and of CTDs-ILDs in Afro-Caribbeans, compared to that in Europeans.

This study, with a comprehensive recruitment and stringent diagnostic criteria, emphasises the importance of secondary ILDs, particularly CTDs-ILDs and the relatively low prevalence of IPF, and confirms that sarcoidosis is a rare disease in France.

This article has supplementary material available from erj.ersjournals.com

Received: Dec 082016 | Accepted after revision: April 262017

Support statement: Funding was provided by the Chancellerie des Universités de Paris (Legs Poix), Paris, France. Funding information for this article has been deposited with the Crossref Funder Registry.

Conflict of interest: Disclosures can be found alongside this article at erj.ersjournals.com

Copyright OERS 2017 
Affiliations: 'Assistance Publique - Hôpitaux de Paris, Service de Pneumologie, Hôpital Avicenne, Bobigny, France. ${ }^{2}$ Université Paris 13, Sorbonne Paris Cité, EA2363 'hypoxie et poumon', Paris, France. ${ }^{3}$ Sorbonne Universités, UPMC Univ Paris 06, INSERM, Pierre Louis Institute of Epidemiology and Public Health (IPLESP UMRS 1136), Epidemiology of Allergic and Respiratory Diseases Department (EPAR), Saint-Antoine Medical School, Paris, France. ${ }^{4}$ Assurance Maladie, DRSM lle de France, Paris, France. ${ }^{5}$ Assistance Publique Hôpitaux de Paris, Service de Radiologie, Hôpital Avicenne, Bobigny, France. ${ }^{6}$ Assistance Publique - Hôpitaux de Paris, Service d'Anatomie Pathologique, Hôpital Avicenne, Bobigny, France. ${ }^{7}$ Service de Pneumologie, Hôpital Robert Ballanger, Aulnay sous-bois, France. ${ }^{8}$ Assistance Publique - Hôpitaux de Paris, Service de Pneumologie, Hôpital Tenon, Paris, France. ${ }^{9}$ Assistance Publique - Hôpitaux de Paris, Service de Pneumologie A, Hôpital Bichat, Paris, France. ${ }^{10}$ Service de Pneumologie, Hôpital de Monfermeil, Montfermeil, France. ${ }^{11}$ Assistance Publique - Hôpitaux de Paris, Service de Médecine Interne, Hôpital Jean Verdier, Bondy, France. ${ }^{12}$ Service de Médecine Interne, Hôpital de Saint-Denis, Saint-Denis, France. ${ }^{13}$ Assistance Publique Hôpitaux de Paris, Service de Médecine Interne, Hôpital Avicenne, Bobigny, France. ${ }^{14}$ Assistance Publique Hôpitaux de Paris, Service de Rhumatologie, Hôpital Avicenne, Bobigny, France. ${ }^{15}$ Sciences PO, Projet ERC Silicosis, Paris, France.

Correspondence: Hilario Nunes, Service de Pneumologie, Hôpital Avicenne, 125 rue de Stalingrad, 93009 Bobigny, France. E-mail: hilario.nunesđaphp.fr

\section{Introduction}

Interstitial lung diseases (ILDs) form a heterogeneous group of disorders that comprise more than 200 entities commonly encountered by general practitioners and physicians of various specialties [1-4]. ILDs are classically divided into four categories: (i) ILDs of known causes, e.g. connective tissue diseases (CTDs)/vasculitis, environmental or occupational, and drug induced; (ii) idiopathic interstitial pneumonias (IIPs); (iii) sarcoidosis; and (iv) other forms of ILDs e.g. lymphangioleiomyomatosis, Langerhans' cell histiocytosis, alveolar lipoproteinosis or eosinophilic pneumonia. Considerable progress has been made over the last two decades in the diagnosis of ILDs. In 2002, an international consensus classification provided harmonised nomenclature and diagnostic criteria for IIPs [5] with an update in 2013 [6]. More specifically, a uniform set of diagnostic criteria for idiopathic pulmonary fibrosis (IPF) was established in 2000 [7] and significantly revised in 2011 [8].

So far, there are few epidemiological studies devoted to the prevalence and incidence of all ILDs, and these have many methodological limitations. Some may suffer from omission of patients followed by non-pulmonologists and other from an uncertainty regarding the precision of diagnoses recorded in the registries and codes from administrative claims. This is evident for IIPs, of which diagnosis can be very difficult and demands a multidisciplinary and expert approach [8]. Last, most published studies predate 2000, and few were published since the 2011 diagnostic criteria for IPF [9-12].

The existing publications show wide ranges in the prevalence and incidence of the various ILDs between countries $[1-4,13]$. These disparities may reflect methodological differences or true genetic or demographic specificities, since the frequency of the majority of ILDs is influenced by age, gender, ethnicity, and smoking habits [14].

The aim of this study was to estimate the prevalence and incidence of ILDs in adults in a French urban multi-ethnic county. In order to achieve completeness of recruitment, we used several sources and called on all specialists who were likely to have cases. To guarantee diagnosis accuracy, cases were centrally discussed by an experienced multidisciplinary panel.

\section{Methods}

\section{Study setting}

An observational study was carried out between January 1 and December 31, 2012 to estimate the prevalence and incidence of chronic ILDs in inhabitants living in Seine-Saint-Denis county, France. The county is part of the highly urbanised and multi-ethnic Greater Parisian agglomeration. It consists of 1,538,726 inhabitants, including 1194601 adults aged $\geqslant 15$ years, with a male-to-female ratio (M/F) of 0.94. Seine-Saint-Denis has a distinctive demographic profile compared to the general French population, younger (mean age: 35.3 versus 40.5 years) and with a higher proportion of people of extra-European origin $(20.7 \%$ versus $4.9 \%)$ (table S1). Information on the demographic structure of the county and the French general population originates from the 2012 nationwide population census [15].

\section{ILD case definition}

Prevalent cases referred to patients alive at the time of study initiation with a known unrecovered ILD, whatever the delay from initial diagnosis. Incident cases referred to patients with a newly diagnosed ILD during the study period. 
Sources of case identification

Two separate sources of patients were used:

Clinical source: hospital and community physicians

Public and private hospitals

31 departments of pulmonology, rheumatology, internal medicine, occupational medicine and geriatrics, representing all the seven public and 12 private hospitals located in Seine-Saint-Denis were invited to take part in the study, as well as the pulmonology departments of four large public hospitals neighbouring the area under consideration. Three participant departments are tertiary centres specialised in rare lung diseases. All staff members were asked to report prospectively the ILD outpatients or inpatients under their care seen at the clinic or the ward during the one-year study period. We assumed that all ILD patients are generally monitored at least once a year. Where applicable, the hospital's computerised discharge system was also interrogated by the medical informatics department. The staff were contacted every month by e-mail or phone.

\section{Community pulmonologists and general practitioners}

A letter was posted every three months to all 14 community-based pulmonologists and 1285 general practitioners working in Seine-Saint-Denis, containing a registration questionnaire. Non-responders to the letters were approached by phone. In addition, an electronic form of the questionnaire could be transferred via internet to a secure address. To improve the rate of attendance, an advertising message was also inserted in the official journal of Seine-Saint-Denis College of Physicians. Besides verification of inclusion criteria, the questionnaire included the date of ILD diagnosis, geographical origin, smoking habits, and, when determined, the aetiology of ILD.

\section{Seine-Saint-Denis Social Security}

In France, ILDs can be recognised as a disease listed in the "30 chronic illnesses" list (Affections Longue Durée 30) that are covered at a $100 \%$ reimbursement basis by French social security. There is no rule regarding ILD severity for applying for this program, as long as patient's condition is considered to be serious, chronic or costly as judged by the general practitioner and approved by the social security medical expert. The beneficiaries of this program are registered in a regularly updated computerised database under the codes of the International Classification of Diseases version 10 (ICDs-10).

A social security physician (CJDN) interrogated the database to identify the patients with one of the ILD codes (table S2). To ensure that patients were still alive with unresolved disease, only those requiring healthcare within the study period were eligible. Subsequently, social security mailed a letter to each patient identified in its database and not already declared by a physician, asking him or her to provide the contact details of his or her general practitioner or referral specialist. All cases for which medical charts could be further obtained were reviewed.

\section{Inclusion and exclusion criteria}

Inclusion criteria were: (i) age $\geqslant 15$ years at the time of ILD diagnosis; (ii) diffuse infiltration of lung parenchyma at chest radiography or HRCT; (iii) duration of symptoms $>2$ months or clinical latency; (iv) residence in Seine-Saint-Denis during the study period. HIV patients were excluded, as well as ILDs due to neoplastic diseases, infections, and congestive heart failure.

\section{Confirmation of aetiological diagnoses}

Three clinicians (BD, HN, and DV), two radiologists (PYB, and $\mathrm{MB}$ ), and one pathologist (MK) discussed each case, after a systematic review of medical charts, HRCT and surgical lung biopsy (SLB), when applicable. The aetiology of ILD was considered "determined" when a diagnosis could be attributed in consensus according to the current guidelines, including 2011 ATS/ERS/JRS/ALAT diagnostic criteria for IPF [8]. The aetiology of ILD remained "undetermined" when a diagnosis could not be established on the basis of available investigations after multidisciplinary discussion (MDD).

For unreviewable cases, information on the aetiological diagnosis was taken from the completed questionnaires or the ICDs-10 codes.

\section{Ethics}

The study was approved by the local ethics committee (Comité de Protection des Personnes Ile-de-France) and by the French data protection authority $\left(n^{\circ} \mathrm{DR}-2010\right.$-027). A signed written consent was required for patients identified by social security. 


\section{Statistical analyses}

The crude prevalence was estimated as the ratio of the number of current cases (new and pre-existing) over the considered period to the at-risk population during the given period. The incidence was estimated as the ratio of new cases during the considered period to the at-risk population during the given period. The confidence intervals were calculated by using the asymptotic Wald test. Standardised incidence and prevalence rates of each ILD for the geographical origin groups in the county were estimated. Due to the small sample size, additional standardisation on age and gender was not conducted. Multinomial logistic regression analysis was carried out in order to predict the probabilities of the different ILDs [16, 17]. This estimation procedure allowed us to estimate the odds of each ILD according to each geographical origin group and relative to the referent group (European) after controlling for sex, age and smoking habits as confounders. A Bonferroni's adjustment was used to account for multiple comparisons. The software package used for the statistical analysis was STATA (StataCorp.2013. Stata Statistical Software: Release 13. College Station, TX: StataCorp LP).

\section{Results}

\section{Crossover analysis}

Figure 1 shows the flow diagram of case identification. 27 of the 31 participating hospital departments declared at least one case. The rate of response was $100 \%$ for the community pulmonologists and $28.5 \%$ $(366 / 1285)$ for the general practitioners. As indicated in figure 2, contributions from the sources were 831 patients for clinicians (public and private hospitals, community pulmonologists and general practitioners) after exclusion of 58 duplicates, and 588 for social security. Among the cases reported by social security, 249 (42.3\%) were duplicates with the clinical source and 339 cases were identified only by social security. A letter was sent to these 339 patients, of which 118 (34.8\%) responded. The total number of included cases was 1170, which was obtained by summing the clinical and social security sources without duplicates. Among these 1170 cases, the charts of 848 (72.5\%) could be obtained for central reviewing, including $802 / 831$ cases (96.5\%) from the clinical source, and 46 from the social security source $(13.6 \%$ of 339 cases). The characteristics of the reviewed population are summarised in table 1.

\section{Prevalence and incidence of ILDs}

The estimated overall crude prevalence and incidence were 97.9 per 100.000 and 19.4 per 100.000 per year, respectively.

\section{Prevalence}

\section{Reviewed population}

The aetiology of ILD was determined for 788/848 (92.9\%) patients, and remained undetermined after MDD for $60(7.1 \%)$. As illustrated in figure 3, the underlying categories of ILDs were as follows: sarcoidosis: 361 cases (42\%); ILDs of known causes: 260 cases (31\%); IIPs: 145 cases (17\%); and other forms of ILDs: 22 cases (3\%). The most frequent disease was sarcoidosis, followed in decreasing order by

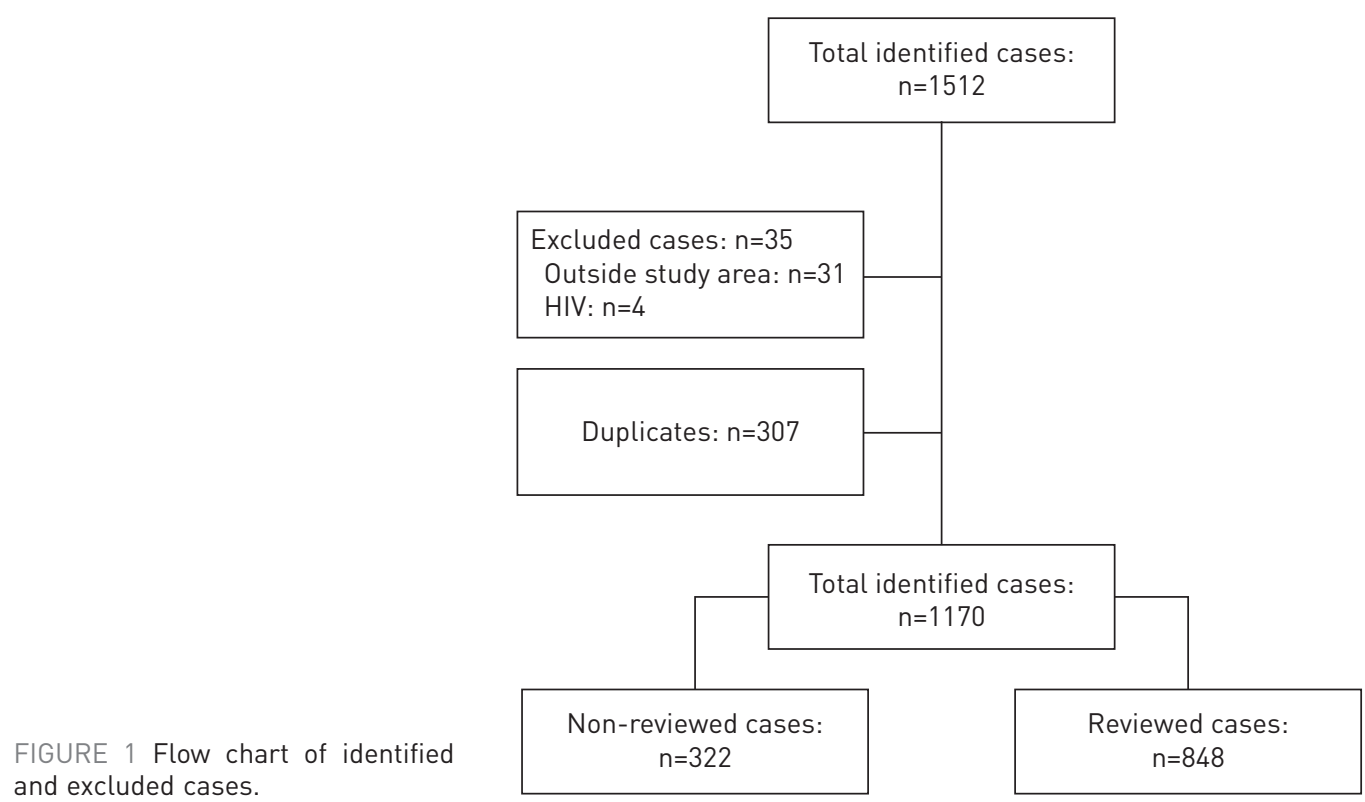




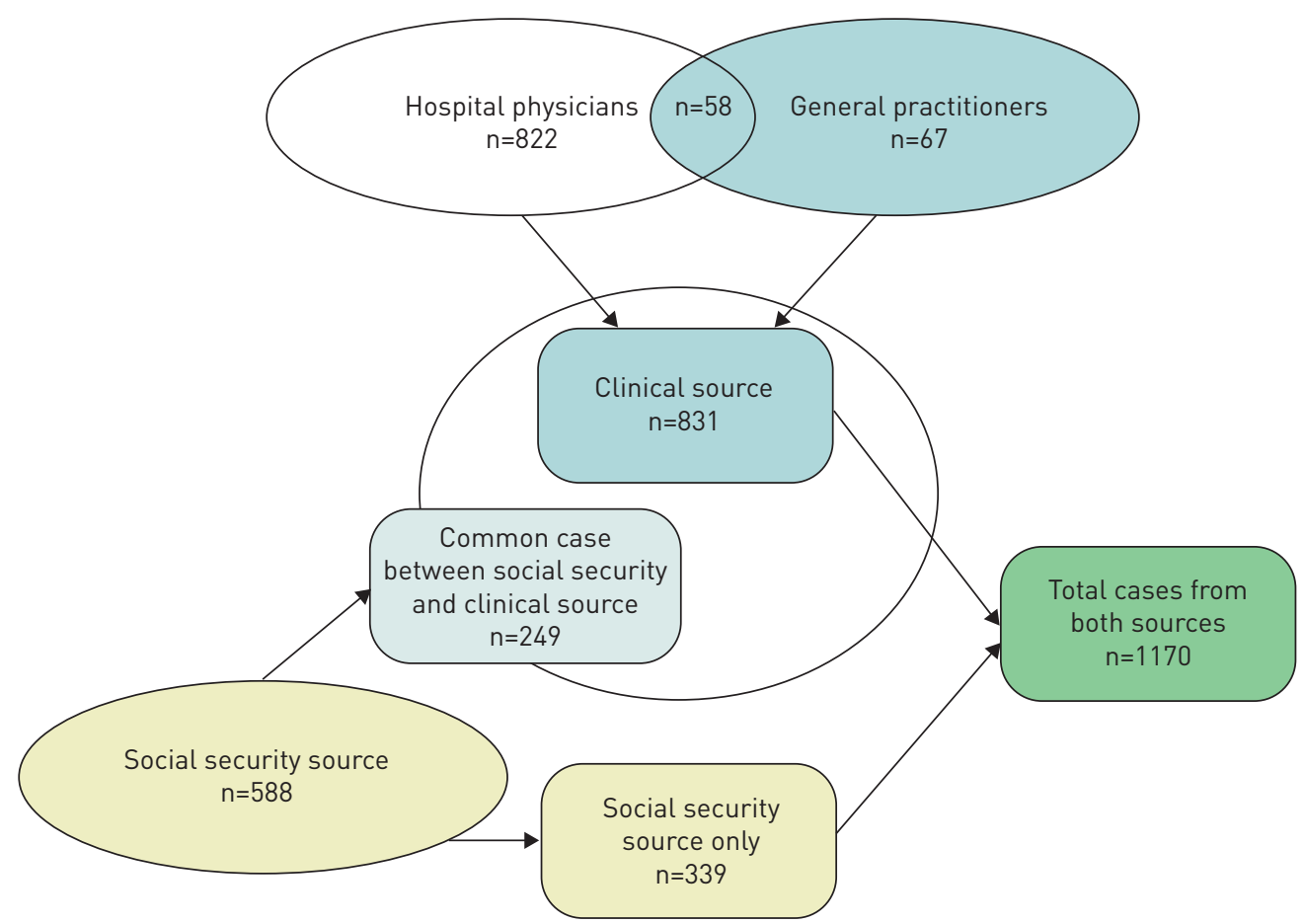

FIGURE 2 Crossover analysis of sources of cases.

connective tissue diseases/vasculitis associated ILDs (CTDs-ILDs) ( $\mathrm{n}=145,17 \%)$, IPF ( $\mathrm{n}=98,11 \%)$, occupational ILD $(n=42,5 \%)$, drug-induced ILD ( $n=31,3 \%)$, hypersensitivity pneumonitis (HP) $(n=28$, $3 \%)$, and idiopathic nonspecific interstitial pneumonia (NSIP) $(n=20,2 \%)$. The most commonly involved CTDs were primary Sjögren's syndrome $(n=40)$, and rheumatoid arthritis $(n=33)$. Incriminated drugs were diverse, the most frequent being amiodarone $(n=10)$. The most frequent HP was bird fancier's lung $(\mathrm{n}=13)$. The most frequent pneumoconiosis was silicosis $(\mathrm{n}=21)$.

Considering the 60 patients with undetermined diagnosis, ILD was unclassifiable despite SLB in six. Two diagnoses were suggested in 53 patients, three in six patients, and four diagnoses in one patient. The majority of cases had a diagnostic uncertainty between IPF and idiopathic NSIP $(n=34)$. The gamut of

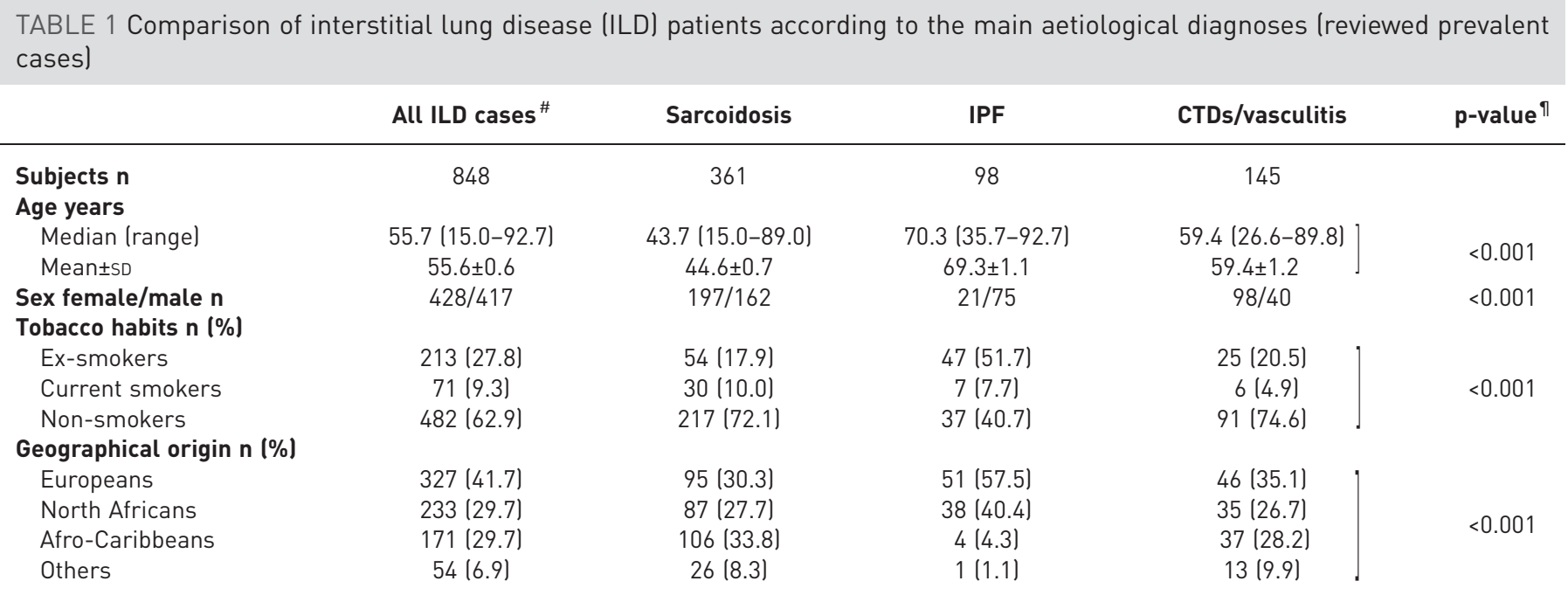

IPF: idiopathic pulmonary fibrosis; CTDs: connective tissue diseases. ${ }^{\#}$ : information regarding sex, tobacco habits and geographic origin was not available in 3, 82 and 63 cases, respectively; " comparison between sarcoidosis, IPF and CTDs/vasculitis-associated ILDs. 
a)

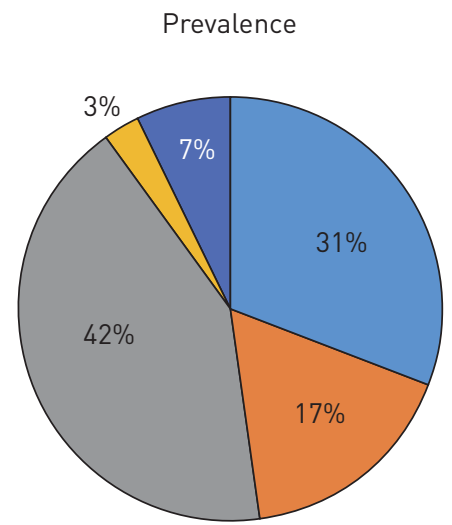

ILDs of known causes

Sarcoidosis

Undetermined

c)

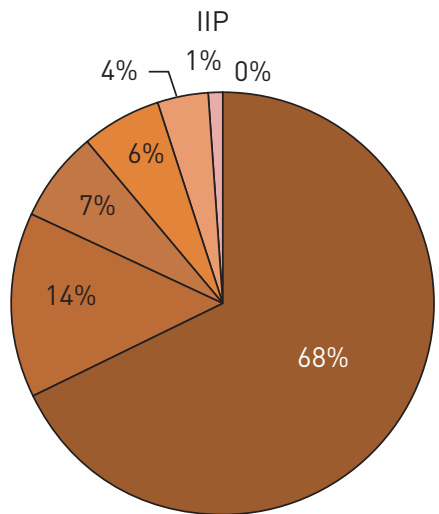

IPF

NSIP

Unclassified
Particular ILDs b) ILDs of known causes

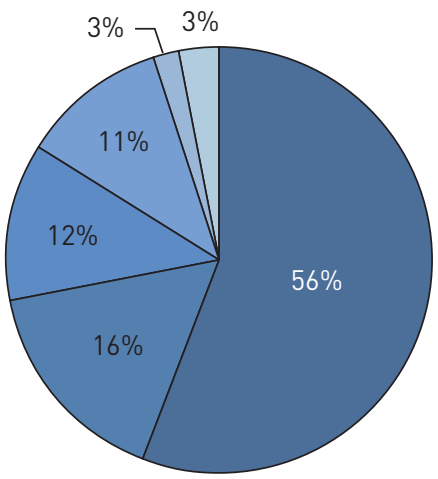

CTDs/vasculitis ILDs

Pneumoconioses

Drug-induced

$\square \mathrm{HP}$

Radiation-induced

d) Particular ILDs

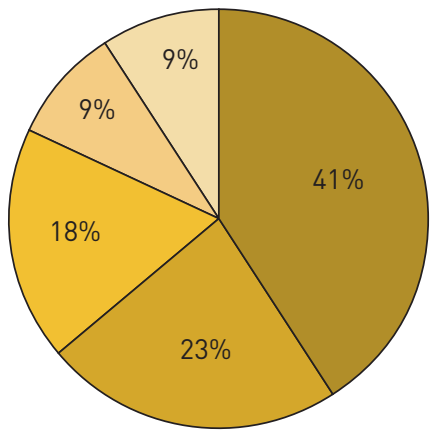

LAM $\square$ CIEP $\square$ PLCH $\square$ PAP $\square$ Others

FIGURE 3 Distribution of prevalent interstitial lung disease (ILD) cases according to underlying aetiological diagnoses (reviewed cases). IIPs: idiopathic interstitial pneumonias; CTDs: connective tissue diseases; HP: hypersensitivity pneumonitis; IPF: idiopathic pulmonary fibrosis; NSIP: nonspecific interstitial pneumonia; DIP: desquamative interstitial pneumonia; COP: cryptogenic organising pneumonia; RBILD: respiratory bronchiolitis with ILD; LIP: lymphoid interstitial pneumonia; LAM: lymphangioleiomyomatosis; CIEP: chronic idiopathic eosinophilic pneumonia; PLCH: pulmonary Langerhans' cell histiocytosis; PAP: pulmonary alveolar proteinosis.

differential diagnoses included IPF ( $n=39)$, NSIP $(n=46)$, HP $(n=15)$, CTDs-ILDs $(n=5)$, pneumoconiosis $(n=3)$, sarcoidosis $(n=6)$, drug-induced $(n=5)$ and other $(n=10)$.

Estimates of the prevalence of the crude ILDs are indicated in table 2. The crude prevalence of sarcoidosis and CTDs-ILDs was 30.22 per 100.000 and 12.14 per 100.000 , respectively. The crude prevalence of IPF was 8.20 per 100.000 and that of idiopathic NSIP was 1.67 per 100.000 . The prevalence of fibrotic IIPs (IPF, idiopathic NSIP and undetermined diagnosis between IPF and idiopathic NSIP) reached 12.63 per 100.000 .

Age and sex-standardised prevalence rates are shown in figure 4 and tables S3 and S4. Patients' characteristics for the main diagnoses (sarcoidosis, IPF and CTDs-ILDs) are summarised in table 1. Aetiological groups were significantly different for age at diagnosis $(p<0.001)$, sex ratio $(p<0.001)$, geographical origin $(\mathrm{p}<0.001)$, and smoking habits $(\mathrm{p}<0.001)$.

\section{Whole cohort}

Considering the total 1170 prevalent cases, the aetiology could be determined for 1095 patients (93.6\%), based on the MDD for reviewed cases $(n=788)$, and, for unreviewable cases, on the questionnaires $(n=14)$, and the ICDs-10 codes $(n=293)$. The repartition of diagnoses was generally similar to that found in the 
TABLE 2 Crude prevalence and incidence of interstitial lung diseases (ILDs) in Seine-Saint-Denis (reviewed cases)

\begin{tabular}{|c|c|c|c|c|}
\hline \multirow[t]{2}{*}{ ILD cases } & \multicolumn{4}{|c|}{ Population $>15$ years of age ${ }^{\#}$} \\
\hline & $\begin{array}{c}\text { Subjects } \\
n\end{array}$ & $\begin{array}{l}\text { Prevalence } \\
\text { per } 100000\end{array}$ & $\begin{array}{c}\text { Subjects } \\
n\end{array}$ & $\begin{array}{l}\text { Incidence per } \\
100000 \text { per year }\end{array}$ \\
\hline All identified cases & 1170 & 97.9 & 232 & 19.4 \\
\hline Reviewed cases & 848 & 71.0 & 219 & 18.3 \\
\hline ILDs of known cause & 260 & 21.8 & 77 & 6.5 \\
\hline CTDs/vasculitis & 145 & 12.1 & 39 & 3.3 \\
\hline Pneumoconioses & 42 & 3.5 & 9 & 0.8 \\
\hline Drug-induced ILDs & 31 & 2.6 & 14 & 1.2 \\
\hline $\mathrm{HP}$ & 28 & 2.3 & 11 & 0.9 \\
\hline Radiation-induced pneumonitis & 7 & 0.6 & 1 & 0.1 \\
\hline Others" & 7 & 0.6 & 2 & $0.3 \S$ \\
\hline IIPs & 145 & 12.14 & 52 & 4.4 \\
\hline IPF & 98 & 8.2 & 33 & 2.8 \\
\hline NSIP & 20 & 1.7 & 10 & 0.8 \\
\hline Desquamative interstitial pneumonia & 10 & 0.8 & 3 & 0.3 \\
\hline Organising pneumonia & 9 & 0.8 & 1 & 0.1 \\
\hline Unclassified (despite SLB) & 6 & 0.5 & 5 & 0.4 \\
\hline RBILD & 2 & 0.2 & 0 & 0.0 \\
\hline LIP & 0 & 0.0 & 0 & 0.0 \\
\hline Sarcoidosis & 361 & 30.2 & 58 & 4.9 \\
\hline Particular ILDs & 22 & 1.8 & 10 & 0.8 \\
\hline LAM & 9 & 0.8 & 4 & 0.3 \\
\hline CIEP & 5 & 0.4 & 1 & 0.1 \\
\hline $\mathrm{PLCH}$ & 4 & 0.3 & 2 & 0.2 \\
\hline PAP & 2 & 0.2 & 1 & 0.1 \\
\hline Others ${ }^{+}$ & 2 & 0.2 & 2 & 0.1 \\
\hline Undetermined diagnosis & 60 & 5.0 & 22 & 1.8 \\
\hline Differential diagnosis between IPF and NSIP & 34 & 2.9 & 13 & 1.1 \\
\hline \multicolumn{5}{|c|}{$\begin{array}{l}\text { CTDs: connective tissue diseases; HP: hypersensitivity pneumonitis; IIPs: idiopathic interstitial } \\
\text { pneumonias; IPF: idiopathic pulmonary fibrosis; NSIP: nonspecific interstitial pneumonia; SLB: surgical } \\
\text { lung biopsy; RBILD: respiratory bronchiolitis with ILD; LIP: lymphoid interstitial pneumonia; LAM: } \\
\text { lymphangioleiomyomatosis; CIEP: chronic idiopathic eosinophilic pneumonia; PLCH: pulmonary } \\
\text { Langerhans' cell histiocytosis; PAP: pulmonary alveolar proteinosis. \#: } 1194601 \text { inhabitants; ?: including } \\
\text { chronic inflammatory colitis ( } n=4) \text {, amyloidosis ( } n=1) \text {, immunoglobulin G4 syndrome (n=1) and progeria } \\
(n=1) ;{ }^{+} \text {: including alveolar microlithiasis }(n=1) \text { and Erdheim Chester's disease (n=1). }\end{array}$} \\
\hline
\end{tabular}

reviewed population (figure S1). The crude prevalence increased to 44.78 per 100.000 for sarcoidosis, 13.14 per 100.000 for CTDs-ILDs, and 11.3 per 100.000 for IPF. The prevalence of what could be realistically considered as fibrotic IIPs was 16.34 per 100.000, when cases with IPF, idiopathic NSIP, undetermined diagnosis between IPF and idiopathic NSIP, and cases registered with J84.1 code were merged (table S5).

Incidence

219 of the 232 incident cases (94.4\%) could be reviewed. The aetiology of ILD was determined in 198 patients and undetermined in $29(13 \%)$. As illustrated in figure 5, sarcoidosis represented the higher proportion of new cases $(n=58,26.5 \%)$, followed in decreasing order by CTDs-ILDs $(n=39,17.8 \%)$, IPF $(\mathrm{n}=33,15.1 \%)$, drug-induced ILD $(\mathrm{n}=14,6.4 \%)$, idiopathic NSIP $(\mathrm{n}=10,4.6 \%), \operatorname{HP}(\mathrm{n}=11,5.0 \%)$, and occupational ILD ( $n=9,4.1 \%)$. Estimates of the crude ILDs incidence are presented in table 1 . The crude incidence of sarcoidosis, CTDs-ILDs and IPF was 4.86, 3.26 and 2.76 per 100.000 per year, respectively.

\section{Geographical origin}

As regards the geographical origin-standardised prevalence and incidence rates, sarcoidosis and CTDs-ILDs were more frequent in people from North Africa (i.e. Algeria, Tunisia and Morocco) (60.0 and 26.9 per 100000, respectively) and in Afro-Caribbeans (112.0 and 41.1 per 100000, respectively) than in Europeans (10.7 and 5.7 per 100000, respectively) (Supplementary material, Table S6). Interestingly, the origin-standardised prevalence rate of IPF was higher in North Africans than in Europeans and Afro-Caribbeans (26.9 and 5.8 per $100000,4.2$ per 100000, respectively). The same trends were observed 

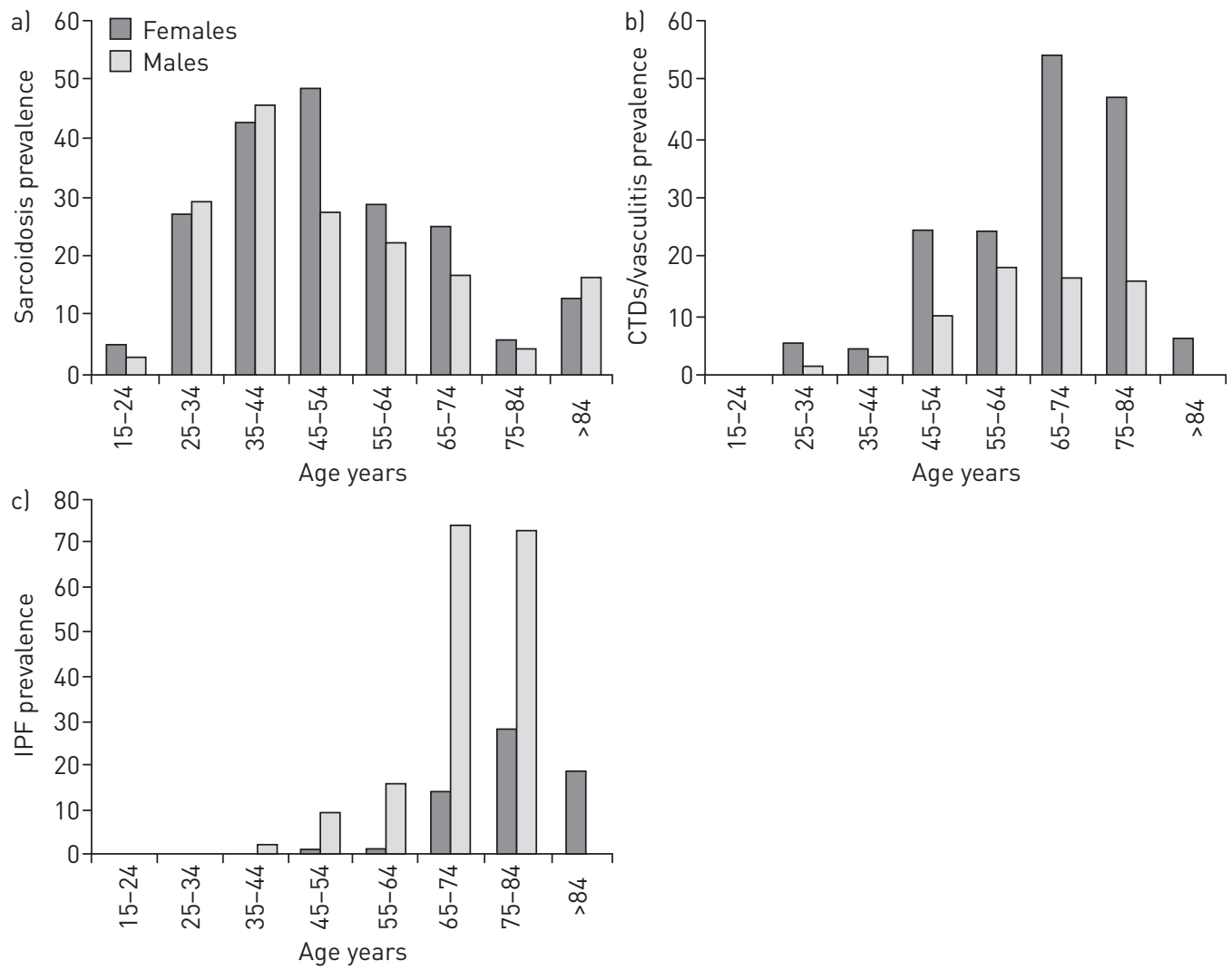

FIGURE 4 Distribution of prevalence rates according to sex and age for the main aetiological diagnoses (reviewed cases). CTDs: connective tissue diseases; IPF: idiopathic pulmonary fibrosis.

for incidence. In addition, the adjusted multinomial model in ILD patients demonstrated a significantly increased risk of sarcoidosis in Afro-Caribbeans (OR: 2.9, 95\% CI [1.6-4.5]) and North Africans (OR: 1.8, 95\% CI [1.8-3.0]), a significantly increased risk of CTDs-ILDs in Afro-Caribbeans (OR: 4.4, 95\%CI [2.28.7]) and a trend for North Africans (OR: 1.7, 95\% CI [1.0-3.0]) as compared to Europeans (table 3).

\section{Discussion}

Our study identified 1170 ILD cases corresponding to a crude overall prevalence of 97.9 per 100000 per year and incidence of 19.4 per 100000 per year. In the 848 cases that could be reviewed and confirmed, the most prevalent diagnoses were sarcoidosis (30.2 per 100000), CTDs-ILDs (12.1 per 100000), and IPF (8.2 per 100000). The crude prevalence and incidence of ILDs appear to be within the most elevated estimates published. Whereas the rates of IPF are lower than previously stated, those of ILDs of known causes are higher, in particular CTDs-ILDs and occupational ILDs. Although sarcoidosis is the most represented disease, accounting for $42 \%$ of cases, its prevalence is in the range that defines rare disorders.

Overall, the crude prevalence of ILDs in our study is even greater than that reported by COULTAS (67-81 per 100000 in the US) [1] and the incidence is close to the highest estimates (25.8 per 100000 per year in Turkey) [4]. Two original findings are of particular interest. First, ILDs due to a recognised cause were one of the predominant diagnoses, just behind sarcoidosis. Among these secondary ILDs, CTDs were the most prominent, even more than IPF, accounting for $16.0 \%$ and $17.8 \%$ of prevalent and incident reviewed cases, respectively. The crude prevalence was 12.1 per 100000 . In previously published studies, CTDs represent $8.9 \%-14.8 \%$ of ILDs $[14,18,19,3]$, with an estimation of prevalence of 2.39 per 100000 in Greece [3]. This result may be related to the involvement of physicians of various specialties, including not only pulmonologists but also rheumatologists and internal medicine physicians. In fact, some CTDs patients have a subclinical ILD that is marginal or overshadowed by the more preoccupying co-existent peripheral symptoms, and are not necessarily referred to a pulmonologist. In addition, international guidelines on IIPs $[6,8]$ and recent studies [20] have raised pulmonologists' awareness of the need to search carefully for CTDs signs and perform autoimmune serologies in patients with ILDs. As regards pneumoconioses, their fairly high contribution may result from the rich industrial history of Saint-Denis county, the involvement of occupational specialists and social security. The environmental causes are important to investigate, as confirmed by the high prevalence of HP in the recent study from SINGH [21]. 
a)

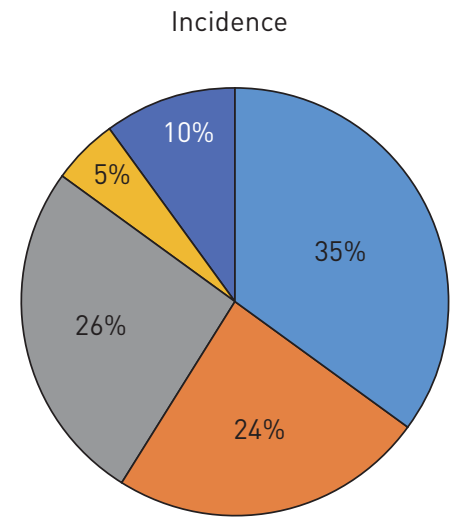

ILDs of known causes

Sarcoidosis

Undetermined

c)

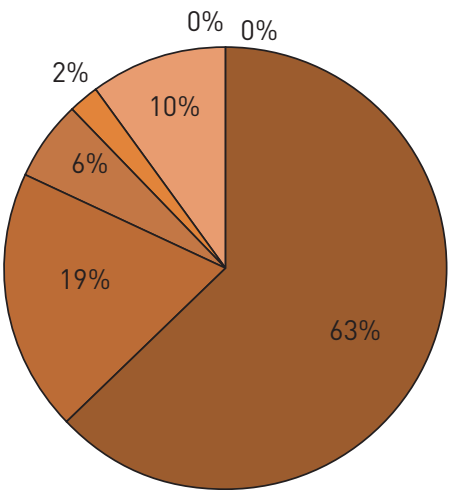

$\square$ IPF $\square$ NSIP $\square$ DIP $\square$ COPD

Unclassified

RBILD b)

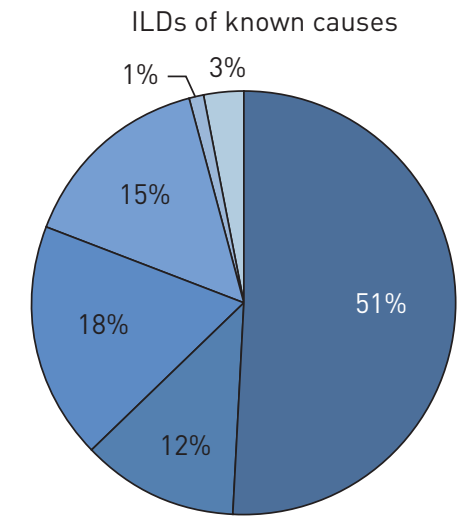

CTDs/vasculitis ILDs

Pneumoconioses

Drug-induced

$\square \mathrm{HP}$

Radiation-induced pneumonitis

d)

Particular ILDs

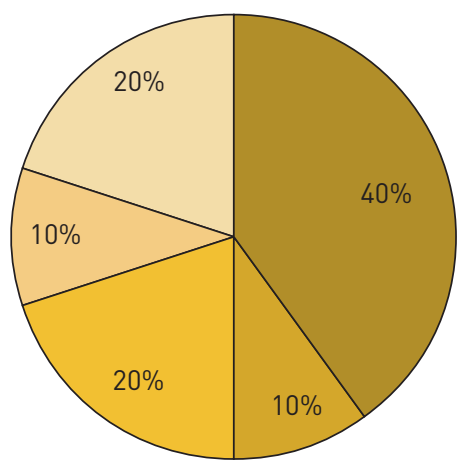

$\square$ LAM $\square$ CIEP $\square$ PLCH $\square$ PAP $\square$ Others

FIGURE 5 Distribution of incident interstitial lung disease (ILD) cases according to underlying aetiological diagnoses (reviewed cases). IIP: idiopathic interstitial pneumonia; CTDs: connective tissue diseases; HP: hypersensitivity pneumonitis; NSIP: nonspecific interstitial pneumonia; DIP: desquamative interstitial pneumonia; COPD: chronic obstructive pulmonary disease; RBILD: respiratory bronchiolitis with ILD; LIP: lymphoid interstitial pneumonia; LAM: lymphangioleiomyomatosis; CIEP: chronic idiopathic eosinophilic pneumonia; PLCH: pulmonary Langerhans' cell histiocytosis; PAP: pulmonary alveolar proteinosis.

Another point is the relatively low frequency of IPF, with a crude prevalence of 8.20 per 100000 and incidence of 2.76 per 100000 per year, which may be explained by the strict application of 2011 ATS/ERS/ JRS/ALAT diagnostic criteria. It is also probable that many cases previously labelled as IPF are now reclassified as ILDs secondary to CTDs or pneumoconiosis. Moreover, the large majority of published studies have no reviewing process or MDD, and use the J84.1 ICDs code ("Other interstitial pulmonary diseases with fibrosis") for the broad definition of IPF; a narrow definition requires further diagnostic testing prior to IPF diagnosis [10, 11, 22-26]. This code does not distinguish IPF and idiopathic NSIP. By contrast, the two entities are clearly separated in our work. When IPF, idiopathic NSIP, and cases with a strong suspicion of IPF or idiopathic NSIP were gathered, that is fibrotic IIPs, the prevalence rose to 12.63 per 100000 and incidence to 4.69 per 100000 per year. These results are lower than in studies based on registries (prevalence and incidence of 25.6 per 100000 and 7.5 per 100000 per year in Italy [27]; prevalence and incidence of 20.0 per 100000 and 9.0 per 100000 per year in Canada [11]), with the exception of a very recent one (prevalence of 2.3 per 100.000 in Italy [10]). They are however higher than in most studies with declared cases (prevalence of 1.25 and $3.38 / 10^{5}$, and incidence of 0.22 and 0.93 per 100.000 per year in Belgium [19] and in Greece [3], respectively). For incidence, published ranges are between 3 and 9 per 100000 per year for Europe and North America [28, 29]. 
TABLE 3 Odds ratios (95\% confidence intervals) according to geographical origin for the main aetiological diagnoses (multinomial logistic regression analysis, reviewed cases)

\begin{tabular}{|c|c|c|c|c|}
\hline \multirow[t]{2}{*}{ Diagnosis } & \multicolumn{4}{|c|}{ Geographical origin } \\
\hline & Europeans & Afro-Caribbeans & North Africans & Others \\
\hline \multicolumn{5}{|l|}{ Sarcoidosis } \\
\hline OR $(95 \%$ CI) & 1 & $2.972(1.615-5.468)$ & $1.822(1.118-2.970)$ & $1.727(0.792-3.769)$ \\
\hline p-value & NA & $<0.001$ & 0.016 & 0.170 \\
\hline \multicolumn{5}{|l|}{ IPF } \\
\hline OR $(95 \% \mathrm{CI})$ & 1 & $0.604(0.163-2.241)$ & $1.418(0.824-2.440)$ & $0.288(0.036-2.303)$ \\
\hline$p$-value & NA & 0.451 & 0.208 & 0.240 \\
\hline \multicolumn{5}{|c|}{ CTDs/vasculitis ILDs } \\
\hline OR $(95 \% \mathrm{Cl})$ & 1 & $4.383(2.210-8.693)$ & $1.767(1.032-3.027)$ & $2.191(0.931-5.157)$ \\
\hline p-value & NA & $<0.001$ & 0.038 & 0.073 \\
\hline
\end{tabular}

IPF: idiopathic pulmonary fibrosis; CTDs: connective tissue diseases; ILDs: interstitial lung diseases.

The relatively low frequency of IPF may also be, at least in part, explained by the demographic structure of Seine-Saint-Denis county, which has a young population [15]. In fact, when focusing on older population, the standardised prevalence and incidence of IPF augmented to 21.4 per 100000 and 7.5 per 100000 per year in patients over 50 years-old, 39.0 per 100000 and 16.0 per 100000 per year for the in-patients over 65 years-old (table S4). These results are quite similar to those of Fernandez Perez et al. (prevalence of 27.9 per 100.000 and incidence of 8.8 per 100.000 per year in subjects older than 50 years)[22]. They are close to those of Raghu et al. in 2001 but intriguingly much lower than those observed in 2011 (prevalence of 30.5 per 100000 in 2001 and 145.7 per 100000 in 2011; incidence of 15.9 per 100000 per year in 2001 and 31.1 per 100000 per year in 2011 in subjects older than 65 years) [18, 30]. Both studies have been conducted in the US.

Importantly, despite sarcoidosis ranking first among ILDs, it remains a rare disease with a prevalence of 30.22 per 100000 , based on reviewed cases, and with a maximum of 44.78 per 100000 with the addition of completed questionnaires and D.86 ICDs code. This is less than the definition of rare disease $(<66$ per 100000 in the US [31], and 50 per 100000 in Europe [32]). Importantly, D.86 includes all sarcoidosis cases, whatever organ involvement. Indeed, respiratory involvement, which is present in more than $90 \%$ of sarcoidosis patients, could not be ruled out for unreviewable social security cases. Our results are close to those recently published in the UK (incidence of 5 per 100000 per year) [28] and the US (8.8 per 100000 per year for incidence and 60.2 per 100000 for prevalence) [33]. The recently published study by ARKEMA found a higher prevalence and incidence of 160 per 100000 and 11.5 per 100000 per year, respectively [34]. These results emphasise the impact of population and geographical specificities.

Very little data are available concerning the impact of ethnicity on IIPs. It has been suggested that Blacks die of IPF less frequently and Hispanics more frequently than Caucasians [35]. In our study, Afro-Caribbeans had a higher prevalence of CTDs-ILDs and sarcoidosis than Europeans. Interestingly, people from North Africa, who make up the largest non-European group in France, particularly in the Paris metropolitan area, have an increased risk of the main ILDs, compared to Europeans.

The primary strength of our study is the comprehensiveness of cases reporting using two different procedures: declaration by hospital specialists, community pulmonologists and general practitioners as well as the use of the social security centralised registry. Moreover, all specialists who may be involved in monitoring ILD patients participated in the study. The second strength is the reliability of diagnoses, with a standardised review of each case by a skilled and multidisciplinary team, and the application of the latest diagnostic guidelines. The study has also several limitations. First, not all cases from social security could be reviewed because nominative agreement was obtained only for a minority. Second, only $28.5 \%$ of the general practitioners responded to the survey, which is low but comparable to similar studies [36]. The great majority of cases declared by the general practitioners had also been declared by the specialists ( 58 out of 67; fig. 2). This indicates that the contribution of general practioners was small in terms of additional cases (nine cases) but also suggests that the low response rate had probably a low impact in terms of missing cases. Third, social security could only provide the date of inscription in the program of exemption of co-payment charges but not the precise date of ILD diagnosis, so that the incidence may have been underestimated. Last, some cases identified from the social security database were not picked up clinically. These social security cases may have been followed in hospitals that did not contribute much or were located outside the study area. Furthermore, we cannot exclude the possibility that some of the 
non-reviewed patients had a healed disease, which may have overestimated the prevalence of curable diseases, in particular sarcoidosis. We tried to take into account this problem by restricting the study to only patients who required healthcare during the study period.

\section{Conclusion}

This epidemiological study, one of the largest published on ILDs, with a thorough recruitment and systematic review of diagnoses, emphasises the importance of secondary ILDs, in particular CTDs, the relatively low prevalence of IPF when using stringent diagnostic criteria, and it confirms that sarcoidosis is a rare disease.

\section{Acknowledgements}

The authors would like to thank: S. Abad, J.P. Battesti, L. Belmont, J. Bourcereau, D. Bouvry, C. Bron, F. Caux, K. Chaouche, F. Crockett, G. Dennewald, G. Falgarone, A. Fargelat, E. Fois, O. Freynet, N. Gambier, T. Gille, V. Gounant, F. Guillon, A. Guyot, I. Haussman, A. Herve, A. Izadifar, P. Joudiou, J. Kabbani, A. Lavole, H. Liote, A. Mahr, M. Mathieu, C. Nakad, M. Neuville, D. Penaud, B. Piketty, HB Romeo, D. Sadoun, M. Sitbon, F. Smahi, H. Tandjaoui-Lambiotte, L. Tecucianu, C. Toper, M. Wislez.

The authors' contributions to the study were as follows. Substantial contributions to the conception or design of the work, or the acquisition, analysis or interpretation of data for the work: B. Duchemann, I. Annesi-Maesano, C. Jacobe de Naurois, H. Nunes and D. Valeyre. Drafting the work or revising it critically for important intellectual content: B. Duchemann, I. Annesi-Maesano, C. Jacobe de Naurois, P-Y. Brillet, M. Brauner, M. Kambouchner, S. Sanyal, H. Nunes, P-A. Rosental and D. Valeyre. Final approval of the version submitted for publication: all authors. Acquisition of data: B. Duchemann, S. Huynh, R. Borie, J. Piquet, A. Mekinian, J. Virally, Y. Uzunhan, J.M. Naccache, B. Crestani, O. Fain, F. Lhote, R. Dhote, N. Saidenberg-Kermanac'h, D. Valeyre and H. Nunes. Accountability for all aspects of the work in ensuring that questions related to the accuracy or integrity of any part of the work are appropriately investigated and resolved: B. Duchemann.

\section{References}

1 Coultas DB, Zumwalt RE, Black WC, et al. The epidemiology of interstitial lung diseases. Am J Respir Crit Care Med 1994; 150: 967-972.

2 Demedts M, Wells AU, Antó JM, et al. Interstitial lung diseases: an epidemiological overview. Eur Respir J 2001; 18: $2 s-16 s$.

3 Karakatsani A, Papakosta D, Rapti A, et al. Epidemiology of interstitial lung diseases in Greece. Respir Med 2009; 103: 1122-1129.

4 Musellim B, Okumus G, Uzaslan E, et al. Turkish Interstitial Lung Diseases Group. Epidemiology and distribution of interstitial lung diseases in Turkey. Clin Respir J 2014; 8: 55-62.

5 American Thoracic Society/European Respiratory Society International Multidisciplinary Consensus Classification of the Idiopathic Interstitial Pneumonias. Am J Respir Crit Care Med 2002; 165: 277-304.

6 Travis WD, Costabel U, Hansell DM, et al. An official American Thoracic Society/European Respiratory Society Statement: update of the International Multidisciplinary Classification of the Idiopathic Interstitial Pneumonias. Am J Respir Crit Care Med 2013; 188: 733-748.

7 American Thoracic Society. Idiopathic pulmonary fibrosis: diagnosis and treatment. Am J Respir Crit Care Med 2000; 161: 646-664.

8 Raghu G, Collard HR, Egan JJ, et al. An official ATS/ERS/JRS/ALAT statement: idiopathic pulmonary fibrosis: evidence-based guidelines for diagnosis and management. Am J Respir Crit Care Med 2011; 183: 788-824.

9 Nalysnyk L, Cid-Ruzafa J, Rotella P, et al. Incidence and prevalence of idiopathic pulmonary fibrosis: review of the literature. Eur Respir Rev 2012; 21: 355-361.

10 Harari S, Madotto F, Caminati A, et al. Epidemiology of idiopathic pulmonary fibrosis in Northern Italy. PloS One 2016; 11: e0147072.

11 Hopkins RB, Burke N, Fell C, et al. Epidemiology and survival of idiopathic pulmonary fibrosis from national data in Canada. Eur Respir J 2016; 48: 187-195.

12 Behr J, Kreuter M, Hoeper MM, et al. Management of patients with idiopathic pulmonary fibrosis in clinical practice: the INSIGHTS-IPF registry. Eur Respir J 2015; 46: 186-196.

13 Hyldgaard C, Hilberg O, Muller A, et al. A cohort study of interstitial lung diseases in central Denmark. Respir Med 2014; 108: 793-799.

14 Valeyre D, Duchemann B, Nunes H, et al. Interstitial lung diseases. In: Annesi-Maesano I, Lundbäck B, Viegi G, eds. Respiratory Epidemiology (ERS Monograph). Sheffield, European Respiratory Society, 2014; pp. 79-87.

15 Insee. Populations légales 2013 - 93-Seine-Saint-Denis. http://www.insee.fr/fr/ppp/bases-de-donnees/recensement/ populations-legales/departement.asp?dep $=93 \&$ annee $=2013$.

16 Gordon-Larsen P, McMurray RG, Popkin BM. Adolescent physical activity and inactivity vary by ethnicity: the National Longitudinal Study of Adolescent Health. J Pediatr 1999; 135: 301-306.

17 Radin JM, Hawksworth AW, Kammerer PE, et al. Epidemiology of pathogen-specific respiratory infections among three US populations. PLoS One 2014; 9: e114871.

18 Raghu G, Chen S-Y, Yeh W-S, et al. Idiopathic pulmonary fibrosis in US Medicare beneficiaries aged 65 years and older: incidence, prevalence, and survival, 2001-11. Lancet Respir Med 2014; 2: 566-572.

19 Thomeer M, Demedts M, Vandeurzen K. Registration of interstitial lung diseases by 20 centres of respiratory medicine in Flanders. Acta Clin Belg 2001; 56: 163-172.

20 Fischer A, du Bois R. Interstitial lung disease in connective tissue disorders. Lancet 2012; 380: 689-698.

21 Singh S, Collins BF, Sharma BB, et al. Interstitial lung disease (ILD) in India: results of a prospective registry. Am J Respir Crit Care Med 2017; 195: 801-813. 
22 Fernández Pérez ER, Daniels CE, Schroeder DR, et al. Incidence, prevalence, and clinical course of idiopathic pulmonary fibrosis: a population-based study. Chest 2010; 137: 129-137.

23 Hodgson U, Laitinen T, Tukiainen P. Nationwide prevalence of sporadic and familial idiopathic pulmonary fibrosis: evidence of founder effect among multiplex families in Finland. Thorax 2002; 57: 338-342.

24 Lai C-C, Wang C-Y, Lu H-M, et al. Idiopathic pulmonary fibrosis in Taiwan - a population-based study. Respir Med 2012; 106: 1566-1574.

25 von Plessen C, Bergman B, Andresen O, et al. Palliative chemotherapy beyond three courses conveys no survival or consistent quality-of-life benefits in advanced non-small-cell lung cancer. Br J Cancer 2006; 95: 966-973.

26 Raghu G, Weycker D, Edelsberg J, et al. Incidence and prevalence of idiopathic pulmonary fibrosis. Am J Respir Crit Care Med 2006; 174: 810-816.

27 Agabiti N, Porretta MA, Bauleo L, et al. others. Idiopathic pulmonary fibrosis (IPF) incidence and prevalence in Italy. Sarcoidosis Vasc Diffuse Lung Dis 2014; 31: 191-197.

28 Gribbin J, Hubbard RB, Le Jeune I, et al. Incidence and mortality of idiopathic pulmonary fibrosis and sarcoidosis in the UK. Thorax 2006; 61: 980-985.

29 Hutchinson J, Fogarty A, Hubbard R, et al. Global incidence and mortality of idiopathic pulmonary fibrosis: a systematic review. Eur Respir J 2015; 46: 795-806.

30 Raghu G, Chen S-Y, Hou Q, et al. Incidence and prevalence of idiopathic pulmonary fibrosis in US adults 18 64 years old. Eur Respir J 2016; 48: 179-186.

31 Rare diseases act of 2002, public law 107-280-NOV. [cited 2016 May 10]. https://history.nih.gov/research/ downloads/PL107-280.pdf Date last accessed: May 10, 2016.

32 European Commission Health \& Consumer Protection Directorate-Gene. Useful information of rare diseases from an EU perspective. http://ec.europa.eu/health/ph_information/documents/ev20040705_rd05_en.pdf Date last access: May 10, 2016.

33 Baughman RP, Field S, Costabel U, et al. Sarcoidosis in America. analysis based on health care use. Ann Am Thorac Soc 2016; 13: 1244-1252.

34 Arkema EV, Grunewald J, Kullberg S, et al. Sarcoidosis incidence and prevalence: a nationwide register-based assessment in Sweden. Eur Respir J 2016; 48: 1690-1699.

35 Swigris JJ, Olson AL, Huie TJ, et al. Ethnic and racial differences in the presence of idiopathic pulmonary fibrosis at death. Respir Med 2012; 106: 588-593.

36 Mahr A, Guillevin L, Poissonnet M, et al. Prevalences of polyarteritis nodosa, microscopic polyangiitis, Wegener's granulomatosis, and Churg-Strauss syndrome in a French urban multiethnic population in 2000: a capture-recapture estimate. Arthritis Rheum 2004; 51: 92-99. 